\title{
Krankenhausplanung zwischen Versorgung und Wettbewerb
}

ELKE HUSTER-NOWACK, MATTHIAS GRUHL

Elke Huster-Nowack ist Leiterin der Fachabteilung Versorgungsplanung des Amtes Gesundheit in der Behörde für Gesundheit und Verbraucherschutz der Freien und Hansestadt Hamburg

Dr. med. Matthias Gruhl ist Leiter des Amtes für Gesundheit der Behörde für Gesundheit und Verbraucherschutz der Freien und Hansestadt Hamburg

\author{
Die Länder können mit den bisherigen Mitteln \\ der Krankenhausplanung und der staatlichen \\ Investitionsfinanzierung zu ihrem Sicherstellungsauftrag \\ immer weniger nachkommen. Deshalb muss die \\ staatliche Krankenhausplanung neue Wege hin zu einer \\ Gewährleistungsplanung gehen. Dabei soll mit neuen \\ Kriterien für eine qualitativ gute Krankenhausleistung \\ einerseits erreicht werden, dass die Patientinnen und \\ Patienten bei der Wahl der für sie richtigen Einrichtung \\ unterstützt werden und andererseits die Länder eine \\ Möglichkeit erhalten, verstärkt Qualitätskriterien \\ und deren Erfüllung zu krankenhausplanerischen \\ Entscheidungen heran zu ziehen. Nur durch \\ einen solchen geregelten qualitätsorientierten \\ Wettbewerb kann einer zu starken Merkantilisierung \\ der Krankenhäuser entgegengewirkt werden.
}

\section{Einleitung}

Durchschnittlich betritt jeder Versicherte spätestens alle fünf Jahre ein Krankenhaus als Patient. Dazu sollte er die Kaskade der haus- und fachärztlichen ambulanten Versorgung ergebnislos durchlaufen haben und/oder schwerst oder akut erkrankt sein oder einen Unfall erlitten haben. Der Versicherte hat dabei theoretisch die Wahl zwischen 2.000 Krankenhäusern in Deutschland und kann in einem von ca. 500.000 deutschen Krankenhausbetten landen; bei der Bettendichte nimmt Deutschland im europäischen Vergleich damit eine Spitzenposition ein. Die Krankenhäuser kosten jeden Versicherten pro Monat rd. $5 \%$ seiner Lohnsumme, summiert allein für die gesetzliche Krankenversicherung auf 60 Milliarden Euro pro Jahr, ohne Zuzahlungen der Versicherten und Privatliquidatonen. Der Gesamtumsatz die- ser Megawirtschaft „Krankenhäuser“ ist mit rund einer Million Beschäftigter und einem Umsatz von rd. 70 Milliarden Euro ein bedeutender Wirtschaftszweig im Wachstumsmarkt „Gesundheitswesen“. In vielen Regionen ist das Krankenhaus dabei der größte Arbeitgeber.

Diesen wirtschaftlich bedeutenden Versorgungssektor planerisch zu gestalten und dabei die stationäre Versorgung der Bevölkerung sicherzustellen, obliegt den Ländern. Gesetzliche Grundlage dieser Landeskrankenhauspläne sind das Krankenhausfinanzierungsgesetz (\$ 6 KHG) und die landeseigenen Krankenhausgesetze. Die Länder haben also ein Entscheidungsrecht über die Zulassung eines Krankenhauses bei der Versorgung von stationären und teilstationären Patienten. Sie definieren mit ihrer Krankenhausplanung, wer nach welchen Kriterien des Bedarfs, der Wirtschaftlichkeit und der Leistungsfähigkeit den 
Markteintritt als Plankrankenhaus mit einem staatlichen Versorgungsauftrag und damit auch einen Anspruch auf Erstattung der Behandlungskosten nach \$ 108 des Fünften Buches Sozialgesetzbuch (SGB V) erhält.

Dabei sind die Länder einerseits zwar das öffentliche Regulativ, um einen ruinösen Wettbewerb der Krankenhäuser untereinander und eine zu große Belastung der gesetzlichen Krankenkassen zu verhindern, sind aber andererseits als zuständige Behörde oder zuständiges Ministerium Teil einer Landesregierung, die mit Freude die hochwertigen Arbeitsplätze im Krankenhaus begrüßt und sie als wichtigen regionalen Wirtschaftsfaktor würdigt. Zwar ist die gesetzliche Verpflichtung der Länder zur Krankenhausfinanzierung zu konstatieren, gesamtökonomisch betrachtet werden diese Investitionen durch entsprechende Beschäftigungs- und Steuereinnahmeeffekte aber für das Gemeinwesen überkompensiert.

Kein leichter Stand also für verantwortungsvolle Krankenhausplanung im Spannungsfeld zwischen Politik, Regulation und Wettbewerb, zumal zusätzlich die Ökonomisierung der Krankenhäuser diese zu einer Politik der Expansion der immer neuen Ansprüche an mehr Kapazitäten (Betten und Tagesklinikplätze) und der intensiveren Behandlung veranlasst.

\section{Die Realität der Krankenhauslandschaft}

Der Krankenhaussektor in Deutschland hat sich seit 1991 erheblich verändert. Gab es in 1991 noch 2.411 Krankenhäuser mit 665.565 vollstationären Betten, so waren es in 2010 noch 2.064 mit 502.749 Betten und damit um fast 25 $\%$ geringere vollstationäre Kapazitäten. Kleinere Krankenhäuser wurden zusammengeführt, Krankenhausverbünde gegründet und auch Standorte komplett aufgegeben.

Die Fallzahl ist hingegen seit 1991 um nahezu $24 \%$ auf 18032903 in 2010 gestiegen, die Zahl der Belegungstage um rd. $30 \%$ zurück gegangen.

Die Zahl der Beschäftigten im Krankenhaus lag 2010 mit 1112959 um gerade mal 1 \% höher als in 1991 und hinsichtlich der Zahl der Vollkräfte sogar um 7,3\% geringer als vor 20 Jahren. $\mathrm{Zu}$ berücksichtigen ist dabei, dass die Zah-
Tabelle 1: Eckdaten deutscher Krankenhäuser

\begin{tabular}{|l|c|c|c|}
\hline $\begin{array}{l}\text { Krankenhaussektor } \\
\text { in Deutschland in } \\
1991-2010\end{array}$ & 1991 & 2010 & $\begin{array}{c}\text { Veränderung } \\
1991-2010 \text { in \% }\end{array}$ \\
\hline Krankenhäuser & 2.411 & 2.064 & -14.4 \\
\hline Krankenhausbetten & 665.565 & 502.749 & $-24,5$ \\
\hline $\begin{array}{l}\text { Krankenhausbetten } \\
\text { pro 100.000 Ein- } \\
\text { wohner }\end{array}$ & 832 & 615 & $-26,1$ \\
\hline Beschäftigte insges. & 1.111 .625 & 1.112 .959 & $+1,0$ \\
\hline $\begin{array}{l}\text { Beschäftigte Voll- } \\
\text { kräfte }\end{array}$ & 875.816 & 811.729 & $-7,3$ \\
\hline Fallzahl & 14.576 .613 & 18.032 .903 & $+23,7$ \\
\hline Belegungstage & 204.204 .000 & 142.942 .000 & $-30,0$ \\
\hline Verweildauer & 14,0 Tage & 7,9 Tage & $-43,6$ \\
\hline Bettenauslastung & 84,1 & 77,4 & $-6,7$ \\
\hline
\end{tabular}

Quelle: Statistisches Bundesamt len vor dem Hintergrund der Ausgliederung von Teilbereichen der Krankenhäuser in DienstleistungsGmbH's statt eigener Mitarbeiter nicht unbedingt vergleichbar sind. Darüber hinaus haben sich nicht unerhebliche Veränderungen durch die vielfältige Beschäftigung von Honorar- und Konsiliarärzten in den deutschen Krankenhäusern ergeben, insbesondere nach Inkrafttreten des Vertragsarztrechtsänderungsgesetzes zum 1.1.2007.

$30 \%$ weniger Belegungstage bedeuten nicht unbedingt $30 \%$ weniger Patientinnen und Patienten im Krankenhaus. Verweildauerverkürzung und gestiegene Fallzahlen stehen für eine deutliche Arbeitsverdichtung der Mitarbeiter in den deutschen Krankenhäusern mit einer entsprechend hohen Belastung für die Beschäftigten in allen Bereichen.

Mit den zunehmenden Schwierigkeiten kommunaler Krankenhausträger durch vielfach hohe Investitionsbedarfe bei unwirtschaftlichen Klinikstruktu- ren (Größe der Krankenhäuser, Fachgebietsstruktur, baulicher Zustand) hat sich die Krankenhauslandschaft hinsichtlich der Trägerschaft entsprechend verändert. In großem Umfang wurden kommunale und auch freigemeinnützige Krankenhäuser privatisiert mit allen Konsequenzen für Strukturen und Mitarbeiterschaft.

Besonders deutlich wird die Veränderung der Trägerstruktur in Hamburg. Gab es im Jahr 2000 noch 11 öffentliche Krankenhäuser, so ist die Zahl mit dem Verkauf des Landesbetriebs Krankenhäuser an Asklepios auf zwei Krankenhäuser zurückgegangen. Verblieben sind in kommunaler bzw. staatlicher Trägerschaft nur noch das Universitätsklinikum Hamburg-Eppendorf und das Bundeswehrkrankenhaus Hamburg. Entsprechend ist die Zahl der privaten Plankrankenhäuser von 2 auf 14 gestiegen.

Die Krankenkassen und Krankenhausträger werfen den Ländern vor, sie

\section{Tabelle 2: Veränderte Trägerstrukturen}

\begin{tabular}{|c|c|c|c|}
\hline \multicolumn{4}{|c|}{ Krankenhäuser in Deutschland 1992-2010 } \\
\hline Trägergruppe & 1992 & 2010 & $\begin{array}{l}\text { Veränderung } \\
\text { 1992-2010 }\end{array}$ \\
\hline $\begin{array}{l}\text { Öffentliche } \\
\text { Krankenhäuser }\end{array}$ & 1.100 & 630 & $-43,0 \%$ \\
\hline $\begin{array}{l}\text { Freigemeinnützige } \\
\text { Krankenhäuser }\end{array}$ & 943 & 755 & $-20,0 \%$ \\
\hline $\begin{array}{l}\text { Private Kranken- } \\
\text { häuser }\end{array}$ & 358 & 679 & $+48,0 \%$ \\
\hline
\end{tabular}

Quelle: Statistisches Bundesamt 
würden sich immer mehr aus der gesetzlich vorgegebene Krankenhausfinanzierung zurückziehen: In 2001 waren es noch rund 3,3 Milliarden Euro, in 2011 nur noch rund. 2,7 Milliarden Euro. Hingegen seien im gleichen Zeitraum die Klinikausgaben durch die Krankenkassen um rd. $33 \%$ auf nahezu 60 Milliarden Euro angestiegen. Das Investitionsvolumen für die Plankrankenhäuser differiert zwischen den einzelnen Ländern erheblich. Hamburg ist seit vielen Jahren Spitzenreiter in Sachen Krankenhausinvestitionen. Pro Planbett wurden auch in 2011 bundesweit die meisten Mittel nach dem Krankenhausfinanzierungsgesetz (KHG) investiert. Der Senat der Freien und Hansestadt Hamburg trägt damit der Bedeutung der Gesundheitsregion Hamburg Rechnung und investiert massiv in die Neustrukturierung der Hamburger Krankenhäuser.

\section{Ordnungsfaktor Bedarf}

Zweck des Gesetzes zur wirtschaftlichen Sicherung der Krankenhäuser und zur Regelung der Krankenhauspflegesätze (KHG) ist „die wirtschaftliche Sicherung der Krankenhäuser, um eine bedarfsgerechte Versorgung der Bevölkerung mit leistungsfähigen, eigenverantwortlich wirtschaftenden Krankenhäusern zu gewährleisten und zu sozial tragbaren Pflegesätzen beizutragen“. Zwar stehen die drei Begriffe der Leis-

\section{Es zeichnet sich ab, dass besonders die Gerichte der Bedarfsfeststellung die höchste Priorität einräumen.}

tungsfähigkeit, Wirtschaftlichkeit und des Bedarfs gleichberechtigt im Gesetz, es zeichnet sich aber ab, dass besonders die Gerichte der Bedarfsfeststellung die höchste Priorität einräumen.

Aber wie definiert man Bedarf? D. Roberfroid hat 2009 einen Übersichtsartikel über die verschiedenen Kategorien von Bedarfsermittlung in der medizinischen Versorgung aus wissenschaftlicher Sicht veröffentlicht, in dem er die bisherigen Methoden der Bedarfsplanung mit dem Blick in eine Kristallkugel verglich. Er unterscheidet vier Arten: die simple Orientierung am
Ist und deren Fortschreibung („supply projection approach") ist zwar immer noch der verbreitete Standard, kann aber verbessert werden, wenn man einen nachfragebasierten Ansatz („demand orientated approach“) als Grundlage der Bedarfsplanung nehme. Dieser wird weiter optimiert durch die Einbeziehung von Kenntnissen des Bedarfs an medizinischer Leistung (,need-orientated approach“). Die hochwertigste Stufe der Bedarfsplanung gründet sich auf einem Benchmarkingansatz (,benchmarking approach“), bei dem für die jeweilige Bedarfsermittlung abgesicherte und vergleichbare Erkenntnisse in Anlehnung an das Optimum herangezogen werden.

Soweit die Theorie. Und wie ermitteln die Länder den Bedarf in ihrer Krankenhausbedarfsplanung?

Zuerst einmal: Es gibt keine einheitliche Krankenhausplanung in Deutschland. Sie unterscheidet sich in den 16 Länder zwischen einer einfachen Umsetzung von in der Vergangenheit belegten Betten in den Krankenhausplan bis hin zu optimierten Modellen angelehnt an Demografieentwicklung, Diagnosestatistiken, Auslastung und auch medizinischer Entwicklung und ist insofern auf der Wertigkeitsskala von Roberfroid zwischen „supply“ und bestenfalls „need“ einzuordnen.

Allgemein ist, auch bedingt durch die Rechtsprechung, eine Tendenz von der bettenscharfen öffentlichen Planung hin zu einer stärkeren Rahmenplanung in verschiedenen Ländern feststellbar. Dabei gibt das Land, nach welchem Verfahren auch immer ermittelt, nur noch den Gesamtbedarf an Planbetten pro Disziplin und für den Krankenhausplan zugelassenen Krankenhäusern (ggf. Fachabteilungen der Krankenhäuser) an und überlässt es dann den beiden Partnern der Selbstverwaltung, die Verteilung der Gesamtmenge auf die einzelnen Krankenhäuser und Fachdisziplinen vorzunehmen. Zum Teil werden auch nur noch die Fachgebiete und die Gesamtplanbettenzahl des Krankenhauses angegeben.

Wenn sich dann Länder noch weiter zurückziehen und die Gesamtbettenzahl nicht mehr prognostisch ermitteln, sondern am nachzuvollziehenden Ist orientieren, hätten sich die Länder de facto ganz aus der aktiven Krankenhausplanung zurückgezogen. Da sie aber die Verantwortung für die Sicherstellung behalten, muss sich die Frage aufdrängen, welche alternativen Steuerungsmechanismen sie entwickeln, um ihrem Auftrag gerecht zu werden.

\section{Ordnungsfaktor Wirtschaftlichkeit}

Insofern stellt sich die Frage nach der Wirtschaftlichkeit und Leistungsfähigkeit als Kriterium für eine effiziente und quantitativ gute Versorgung heute mehr denn je.

Aber kann man diese Kriterien wirklich so gut überprüfen, dass sie entscheidend für die Krankenhausplanung genutzt werden? Schon die Wirtschaftlichkeit eines Hauses ist schwer für eine Landesbehörde zu bewerten, da unklar ist, was darunter zu verstehen ist:

- dass die Krankenhäuser keine Verluste schreiben (dann müssten ca. 15-20 \% der deutschen Krankenhäuser schließen);

- effizient mit den Krankenkassen zu verhandeln

- möglichst hohen Gewinn generieren (für den es allerdings keinen Re-Investitionszwang in dem Krankenhaussektor laut Gesetz gibt)?

Eine gesetzliche Grundlage für einen Zwang zur Offenlegung der wirtschaftlichen Situation gegenüber der Krankenhausplanungsbehörde gibt es nicht.

Insofern ist das Kriterium der Wirtschaftlichkeit ein stumpfes Schwert der Krankenhausplanung und wird selten meist nur in Übereinstimmung mit den Krankenhausträgern - genutzt, z.B. um unrentable Häuser zu schließen. Da das Land meist noch Abwicklungskosten zu zahlen hat, ist dies oft besser für den Träger als eine Insolvenz.

\section{Ordnungsfaktor Leistungsfähigkeit}

Zukunftsfähiger ist das Kriterium der Leistungsfähigkeit, beinhaltet dies doch nach gängiger Meinung auch die Dimension der Qualität. Wenn es also möglich ist, die Qualität für bestimmte Fachdisziplinen oder Diagnosen zu definieren, wäre damit die Möglichkeit gegeben, ein gutes Krankenhaus für den 
Krankenhausplan und damit also für die GKV-Patienten zuzulassen. Voraussetzung für ein solches Vorgehen wäre es aber, nicht die Krankenhäuser als Ganzes zu betrachten, sondern nur die jeweiligen Abteilungen bzw. Fachdisziplinen des Krankenhauses. Eine qualitativ hochwertige Gynäkologie ist eben kein Garant für eine gute Innere Medizin. Krankenhausplanung würde dann auf eine Fachdisziplinbezogene und damit stärker detaillierte Krankenhausplanung umgestellt werden.

Die Landeskrankenhausgesetze der Länder enthalten Hinweise zur Qualitätssicherung der Krankenhäuser, die zum Teil konkret, zum Teil noch auszugestalten sind bzw. bundesrechtliche Qualitätsregelungen wiedergeben. Eigenständige Vorgaben sieht das Bremische Krankenhausgesetz vom 12.04.2011 vor, das der Krankenhausplanungsbehörde die Befugnis erteilt, in einer Rechtsverordnung Maßnahmen der Qualitätssicherung zu benennen, die für die Aufnahme eines Krankenhauses in den Krankenhausplan verbindlich sein sollen bzw. Strukturvorgaben für die ärztlich fachliche Leitungen in Anlehnung an die Weiterbildungsordnung der Länder festlegt. Das Krankenhausgestaltungsgesetz des Landes Nordrhein-Westfalen (KHGG NRW) beinhaltet gleichfalls Regelungen zur Qualitätssicherung. Danach soll die Öffentlichkeit unter Beachtung der bundesrechtlichen Bestimmungen auch über Qualitätsmerkmale und -indikatoren informiert werden, über die ein Krankenhaus so zu unterrichten hat, dass Patientinnen und Patienten ein direkter Vergleich zwischen verschiedenen Krankenhäusern möglich wird. Im Vordergrund steht hier die Strukturqualität.

Auch in Hessen sieht das Krankenhausgesetz eine Ermächtigungsnorm für die Festlegung verbindlicher Qualitätsstandards vor. Andere Länder, so bisher auch Hamburg, bilden in unterschiedlicher Weise solche Anforderungen in den Feststellungsbescheiden ab. Thüringen hat ebenfalls Qualitätsvorgaben eingeführt.

Die Krankenhauspläne der Länder enthalten also in unterschiedlichem Maße Qualitätsvorgaben, im Wesentlichen zur Strukturqualität und beschränkt auf ausgewählte Fachgebiete, Schwerpunkte bzw. zugewiesene Aufgaben (Geburtshilfe, Schlaganfallversor- gung, Herzinfarktversorgung, Geriatrie, Pädiatrie/Neonatologie, Tumorzentren, Frührehabilitation, Palliativmedizin). Dabei werden auch die regionalen $\mathrm{Ge}$ gebenheiten berücksichtigt.

Aussagen zur Prozessqualität finden sich in den Krankenhausplänen einzelner Länder (z.B. Schlaganfallversorgung, Kardiologie, Perinatal-

\section{Die Entwicklung effektiver} Verfahren zur Messung und Bewertung von Ergebnisqualität steht erst am Anfang.

versorgung). Die Prozessqualität liegt allerdings im Wesentlichen in der Organisationshoheit der Krankenhäuser. Als ein für die Krankenhausplanung zu nutzendes Kriterium der Prozessqualität können Anforderungen an die Krankenhaushygiene weiterentwickelt werden.

Anforderungen an die Ergebnisqualität werden bisher weder in den Landeskrankenhausgesetzen noch in den Krankenhausplänen getroffen. Die Entwicklung effektiver Verfahren zur Messung und Bewertung von Ergebnisqualität steht derzeit erst am Anfang

Es wäre zu prüfen, ob die von den Medizinischen/Wissenschaftlichen Fachgesellschaften implementierten Konzepte wenn möglich in die Planung der Versorgungsstrukturen integriert werden sollten. Der Stellenwert der Entwicklungen kann jedoch noch nicht abschließend eingeschätzt werden.

Dabei sind die (zukünftigen) Arbeitsergebnisse des Institutes nach $\mathbb{} 137$ a SGB V (AQUA, Institut für angewandte Qualitätsförderung und Forschung im Gesundheitswesen) auf deren Nutzung zu überprüfen. Auch sind verschiedene Ansätze der Qualitätsmessung wie die Behandlungsergebnisse aus dem QSRVerfahren der AOK oder Portale wie die der Schön-Kliniken, der Helios-Kliniken oder „qualitätskliniken.de“ auf ihre Nutzung zu prüfen.

Dieser Weg, die Krankenhausplanung stärker auf die Gewährleistung guter Qualität auszurichten und die Bedarfsfrage stärker den Aushandlungsmechanismen zwischen den Krankenhäusern und den Finanziers, also den Krankenkassen, zu überlassen, beginnt in den Ländern zur Zeit. Dies soll nicht als
Rückzug der Länder aus der Krankenhausverantwortung verstanden werden, sondern eher als Übernahme einer stärkeren Verantwortung für das Wohl des Patienten, dem es in erster Linie um gesicherte Qualität seiner Behandlung geht und weniger um die Zahl der Betten in der jeweiligen Fachabteilung.

Damit werden die Zulassung und der Verbleib im Krankenhausplan als neue regulative Hürde eingeführt und lösen das bisherige Diktum ab, dass da heißt: einmal Plankrankenhaus - immer Plankrankenhaus.

Ein vorzeigbares komplettes neues System einer solchen Krankenhausplanung gibt es allerdings noch nicht. Hamburg hat vor, diesen Weg, die Krankenhausplanung stärker an Qualitätskriterien auszurichten - zeitnah weiterzuentwickeln.

\section{Planung und/oder Wettbewerb}

Idealtypisch wird mit dem skizzierten Weg ein neuer ordnungspolitischer, nämlich qualitätsorientierter Rahmen für die Krankenhausplanung gesetzt. Innerhalb dieses Rahmens von Qualität und der damit verbundenen Transparenz haben die Krankenhäuser ihren Spielraum für einen Wettbewerb untereinander, der sich dann aufgrund der staatlichen Rahmensetzung vorrangig an der Qualität ihrer Leistungen ausrichtet.

Selbstverständlich konkurrieren bereits heute Krankenhäuser besonders in Ballungsgebieten untereinander. Dabei spielt eine gesicherte Qualität der Versorgung allerdings kaum eine Rolle. Zwar zählt der Imagefaktor für jedes Krankenhaus, aber dieser basiert allzu oft auf nicht belegbaren Meinungen, Selbstdarstellung und direkte oder indirekte Einflussnahme auf die (ärztlichen) Einweiser, die mit verschiedenen Möglichkeiten an das Krankenhaus gebunden werden. Sie sind es in der Regel, deren nachhaltige Empfehlung die Belegung, insbesondere bei elektiven Behandlungen deutlicher beeinflusst als die eigenständige Entscheidung des Patienten. Hinzu kommt die große Zahl der Patienten, die den Krankenhäusern als Notfall mit Rettungsmitteln zugeführt werden. Diese haben in der Regel keine Möglichkeit, das behandelnde Krankenhaus auszusuchen. Es herrscht also in einem regionalen Krankenhaus- 
markt mit mehreren Anbietern ein sehr untergründiger Wettbewerb, so nicht ein „Monopolanbieter" ein fachliches oder regionales Alleinstellungsmerkmal hat.

Allerdings kommt ein finanzmarktorientierter Wettbewerb um Rendite, Größe und Marktpenetranz hinzu, der zu Lasten der Trägervielfalt und regionaler versorgungsorientierter Einflussnahme geht.

In der Strategie von großen Krankenhauskonzernen spielen die regionalen Besonderheiten, die Einbindung in eine Versorgungsregion, die Zusammenarbeit mit den örtlichen Akteuren und mit anderen Leistungserbringern, die Weiterentwicklung einer regionalen Gesundheitswirtschaft keine große Rolle. Der Begriff der „Gesundheitsfabriken“ macht die Runde. Es stellt sich die Frage, ab welchem Zeitpunkt, trotz der Bemühungen des Kartellrechts um regionale Wettbewerbsbedingungen und der staatlichen Krankenhausplanung, die Marktmacht einzelner Krankenhausträger so stark ausprägt sein wird, dass die Länder ihre Versorgungsverantwortung gar nicht mehr wahrnehmen können. Das alles spricht für die Notwendigkeit einer stärkeren Transparenz sowie regulativer und qualitativer Rahmensetzung.

\section{Exkurs: Versorgung und Ökonomie in der stationären Psychiatrie}

Ein Beispiel für die einen versorgungspolitischen und krankenhausplanerischen Bedeutungswandel, der auch durch die ökonomische Entwicklung geprägt wird, ist die veränderte Einstellung der deutschen Krankenhausträger zur psychiatrischen Versorgung. Lange galt sie bei den Krankenhausträgern als ungeliebtes Schmuddelkind, das eher das Image eines somatisch orientierten Krankenhauses beschädigte.

Anders als bei der pauschalen Abrechnung über DRGs bei den somatischen Fächern hat die Psychiatrie die Möglichkeiten zur Abrechnung tagesgleicher Pflegesätze bewahrt. Die Verweildauer und damit auch die Einnahmen aus psychiatrischen Patienten werden kaum gedeckelt. Dadurch wurde die Psychiatrie plötzlich als cash-cow von den Krankenhäusern geschätzt und als gern gesehene Disziplin in den Krankenhäusern als unverzichtbar erklärt.

Die starke Zunahme von Psychiatriebetten hat sicherlich mit vielen anderen gesellschaftlichen Phänomenen zu tun, alleine mit objektiven Kriterien kann sie jedoch nicht erklärt werden. Es wird berichtet, dass die Zahl der psychisch Erkrankten absolut in Deutschland nicht steigt, sondern eher eine veränderte Einstellung zur Therapie, von wem immer, zu dieser Entwicklung geführt hat. Schlagzeilen wie „psychisch bedingtes Aussetzen am Arbeitsplatz - Immer mehr Fehltage wegen Burnout und Depression" sind den Medien zu entnehmen. Kaum ein Tag vergeht, an dem nicht irgendeine Studie zum besorgniserregenden Anstieg der Behandlung psychischer Störungen oder Erkrankungen veröffentlicht wird. Ausgebrannt, krank und antriebslos: immer mehr Menschen in Deutschland fehlen wegen psychischer Leiden am Arbeitsplatz. Seit dem Jahr 2000 habe sich der Anteil der Fehltage wegen Burnout oder Depressionen etwa verdoppelt, geht aus einer Anfang Juni 2012 in Berlin veröffentlichten Studie der Bundespsychotherapeutenkammer (BPtK) hervor. Derzeit würden 12,3 Prozent aller betrieblichen Fehltage in Deutschland durch psychische Erkrankungen verursacht.

Psychische Erkrankungen führen laut Studie zu besonders langen Fehlzeiten von durchschnittlich 30 Tagen, Arbeitnehmer mit Depressionen fehlten durchschnittlich sogar 39 Tage. Nach jüngsten Berechnungen der Bundesregierung entstehen den Unternehmen jährlich durch psychische Krankheiten Produktionsausfälle von 26 Milliarden Euro. Damit wächst auch der Druck auf die Kran- bedarf im präventiven und ambulanten Sektor. Eine Beeinflussung der Versorgungsebene durch therapiefremde ökonomische Fehlanreize sollte auf jeden Fall verhindert werden.

\section{Fazit}

Mit den bisherigen Mitteln der (häufig nachvollziehenden) Krankenhausplanung und der staatlichen Investitionsfinanzierung können die Länder ihrem Sicherstellungsauftrag immer weniger nachkommen.

Die ordnungspolitische Rahmensetzung muss sich dahingehend ändern, Kriterien für eine qualitativ gute Krankenhausleistung $\mathrm{zu}$ entwickeln und festzulegen und diese transparent $\mathrm{zu}$ machen. Die staatliche Krankenhausplanung wird zu einer Gewährleistungsplanung weiterentwickelt. Nur bei einem so initiierten qualitätsorientierten Wettbewerb ist es innerhalb von Leitplanken möglich, einer Merkantilisierung der Krankenhäuser entgegen zu wirken.

Gesundheit ist bekanntlich ein teures Gut. Von daher sollte die Gesundheitswirtschaft auch eine Wirtschaft für mehr Gesundheit und nicht nur für mehr Wachstum sein. kenhausplanung, möglichst viele neue stationäre psychiatrische Behandlungsmöglichkeiten zu schaffen. Eine solche vielfältig beeinflusste Entwicklung lässt sich aber keinesfalls durch immer mehr Kankenhausbetten kompensieren. Vielmehr besteht ein erheblicher Handlungs-

\section{Es müssen Kriterien für eine qualitativ gute Krankenhausleistung entwickelt, festgelegt und transparent gemacht werden.}

